\title{
Tele-rehabilitation using in-house wearable ankle rehabilitation robot
}

Prashant K. Jamwal, Shahid Hussain, Nazim Mir-Nasiri, Mergen H. Ghayesh, Sheng Q. Xie

Department of Electrical and Electronical Engineering

\section{Abstract}

This article explores wide-ranging potential of the wearable ankle robot for in-house rehabilitation. The presented robot has been conceptualized following a brief analysis of the existing technologies, systems, and solutions for in-house physical ankle rehabilitation. Configuration design analysis and component selection for ankle robot have been discussed as part of the conceptual design. The complexities of human robot interaction are closely encountered while maneuvering a rehabilitation robot. We present a fuzzy logic-based controller to perform the required robot-assisted ankle rehabilitation treatment. Designs of visual haptic interfaces have also been discussed, which will make the treatment interesting, and the subject will be motivated to exert more and regain lost functions rapidly. The complex nature of web-based communication between user and remotely sitting physiotherapy staff has also been discussed. A high-level software architecture appended with robot ensures user-friendly operations. This software is made up of three important components: patient-related database, graphical user interface (GUI), and a library of exercises creating virtual reality-specifically developed for ankle rehabilitation.

Original language English

Pages (from-to) $\quad 1-10$

Number of pages 10

Journal $\quad$ Assistive Technology

State $\quad$ Accepted/In press - Oct 22016

Jamwal, P. K., Hussain, S., Mir-Nasiri, N., Ghayesh, M. H., \& Xie, S. Q. (2016). Telerehabilitation using in-house wearable ankle rehabilitation robot. Assistive Technology, 1-10. DOI: $10.1080 / 10400435.2016 .1230153$ 